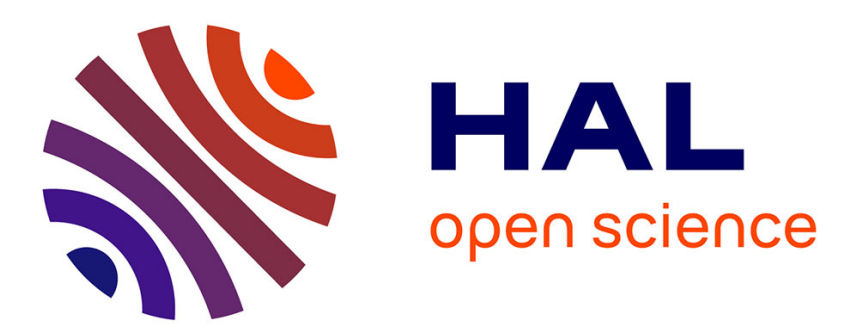

\title{
Particle approximation for first order stochastic partial differential equations
}

\author{
Patrick Florchinger, François Le Gland
}

\section{To cite this version:}

Patrick Florchinger, François Le Gland. Particle approximation for first order stochastic partial differential equations. [Research Report] RR-1502, INRIA. 1991. inria-00075060

\section{HAL Id: inria-00075060 \\ https://hal.inria.fr/inria-00075060}

Submitted on 24 May 2006

HAL is a multi-disciplinary open access archive for the deposit and dissemination of scientific research documents, whether they are published or not. The documents may come from teaching and research institutions in France or abroad, or from public or private research centers.
L'archive ouverte pluridisciplinaire HAL, est destinée au dépôt et à la diffusion de documents scientifiques de niveau recherche, publiés ou non, émanant des établissements d'enseignement et de recherche français ou étrangers, des laboratoires publics ou privés. 


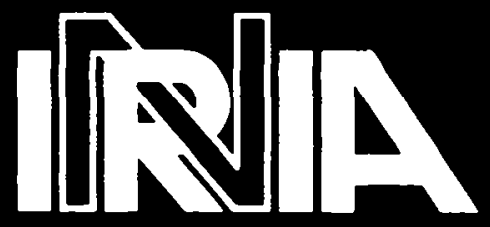

UNITÉ DE RECHERCHE INRIA-SOPHIA ANTIPOLIS

Institut National de Recherche en Informatique et en Automatique

Domaine de Voluceau

Rocquencourt

$$
\text { B.P.105 }
$$

78153 Le Chesnay Cedex

France

Tél.:(1) 39635511

\section{Rapports de Recherche}

$$
\mathrm{N}^{\circ} \mathbf{1 5 0 2}
$$

\section{Programme 5}

Traitement du Signal, Automatique et Productique

\section{PARTICLE APPROXIMATION FOR} FIRST ORDER STOCHASTIC PARTIAL DIFFERENTIAL EQUATIONS

Patrick FLORCHINGER François LE GLAND 


\title{
PARTICLE APPROXIMATION FOR FIRST ORDER STOCHASTIC PARTIAL DIFFERENTIAL EQUATIONS*
}

\author{
Approximation Particulaire pour \\ les Equations aux Dérivées Partielles Stochastiques \\ du Premier Ordre
}

\author{
Patrick FLORCHINGER ${ }^{\S}$ \\ Université de Metz
}

Département de Mathématiques

URA CNRS 399

Ile du Saulcy

F-57045 METZ Cédex

\author{
François LE GLAND \\ INRIA Sophia-Antipolis \\ Route des Lucioles \\ F-06565 VALBONNE Cédex
}

*Presented at : NSF-INRIA Workshop on Applied Stochastic Analysis, Rutgers University, April 29-May 2, 1991. Research partially supported by USACCE under Contract DAJA45-90C-0008.

$\S_{\text {also }}$ : INRIA Lorraine, CESCOM, Technopole de Metz 2000, 4 rue Marconi, F-57070 METZ. 


\begin{abstract}
A class of degenerate second order stochastic PDE is considered, for which a representation result in terms of stochastic characteristics has been proved by Krylov-Rozovskii [2] and Kunita [3,4]. An example of a stochastic PDE in this class has been exhibited in Florchinger-LeGland [1] as the result of a Trotter-like product formula for the Zakai equation of diffusion processes observed in correlated noise. Particle approximations are introduced for this class of stochastic PDE, and error estimates are provided which extend the results of Raviart [6] on first order deterministic PDE.
\end{abstract}

\title{
Résumé
}

On considère une classe d'EDP stochastiques du second ordre dégénérées, pour lesquelles un résultat de représentation à l'aide de courbes caractéristiques stochastiques a été prouvé par Krylov-Rozovskii [2] et Kunita [3,4]. Un exemple d'EDP stochastique de ce type, a été obtenu par Florchinger-LeGland [1] comme résultat d'une formule produit $\dot{a}$ la Trotter pour l'équation de Zakai des processus de diffusion observés dans un bruit corrélé. On étudie des approximations particulaires pour cette classe d'EDP stochastiques, et des estimations d'erreur sont fournies, qui étendent les résultats de Raviart [6] sur les EDP déterministes du premier ordre. 


\section{Introduction}

Consider the following stochastic differential equation

$$
d X_{t}=b\left(X_{t}\right) d t+\sigma\left(X_{t}\right)\left[d W_{t}-e\left(X_{t}\right) d t\right],
$$

where $\left\{W_{t}, t \geq 0\right\}$ is a $d$-dimensional standard Wiener process, and the associated stochastic flow of diffeomorphisms $\left\{\xi_{s, t}(\cdot), 0 \leq s \leq t\right\}$, and define

$$
\begin{aligned}
\Xi_{0, t}(x) \triangleq \exp & \left\{\int_{0}^{t} e^{*}\left(\xi_{0, s}(x)\right) d W_{s}\right. \\
& -\frac{1}{2} \int_{0}^{t} \mid e\left(\left.\xi_{0, s}(x)\right|^{2} d s+\int_{0}^{t} c\left(\xi_{0, s}(x)\right) d s\right\} .
\end{aligned}
$$

Introduce the following partial differential operators

$$
\begin{aligned}
& L \triangleq \frac{1}{2} \sum_{i, j=1}^{m} a^{i, j} \frac{\partial^{2}}{\partial x_{i} \partial x_{j}}+\sum_{i=1}^{m} b^{i} \frac{\partial}{\partial x_{i}}+c, \\
& B_{k} \triangleq e_{k}+\sum_{i=1}^{m} \sigma_{k}^{i} \frac{\partial}{\partial x_{i}}, \quad 1 \leq k \leq d,
\end{aligned}
$$

with $a=\sigma \sigma^{*}$, and the stochastic PDE

$$
d q_{t}=L^{*} q_{t} d t+\sum_{k=1}^{d} B_{k}^{*} q_{t} d W_{t}^{k} .
$$

Because of the relation $a=\sigma \sigma^{*}$ between coefficients of higher order partial derivatives in operators $L$ and $B_{k}$, equation (1.2) is a degenerate second order stochastic PDE or equivalently, after transformation into Stratonovich form, a first order stochastic PDE. Existence and representation results have been obtained by Kunita [4] for (generally nonlinear) first order stochastic PDE, based on the notion of stochastic characteristics.

In a previous work [1], the Zakai equation for the nonlinear filtering of diffusion processes observed in correlated noise has been considered. A decomposition of the Zakai equation has been introduced, exhibiting a degenerate second order stochastic PDE similar to (1.2) in the correction step. In addition, a time discretization scheme has been proposed for this degenerate second order stochastic PDE, with rate of convergence of order $\sqrt{\delta}$, where $\delta$ is the time step.

The purpose of this paper is to provide a discretization scheme of the degenerate second order stochastic PDE (1.2) with respect to the space variable $x \in \mathbf{R}^{m}$. This approximation relies on the representation of the solution in terms of stochastic characteristics, and approximation of the initial condition by a convex linear combination of Dirac masses. This kind of aproximation is called a particle approximation, see Raviart [6]. 
More specifically, for any probability measure $\mu(d x)$ on $\mathbf{R}^{m}$, define the transformed measure $Q_{t} \mu(d x)$ by

$$
\left\langle Q_{t} \mu, \phi\right\rangle=\int \phi\left(\xi_{0, i}(x)\right) \Xi_{0, \imath}(x) \mu(d x),
$$

for any test function $\phi$, or equivalently

$$
Q_{t} \mu(A)=\int_{\varepsilon_{0, \ell}^{-1}(A)} \Xi_{0, t}(x) \mu(d x)
$$

Note that, if $\phi$ is regular enough, then the Itô formula gives

$$
d\left[\phi\left(\xi_{0, t}(x) \Xi_{0, t}(x)\right]=L \phi\left(\xi_{0, t}(x)\right) \cdot \Xi_{0, t}(x) d t+\sum_{k=1}^{d} B_{k} \phi\left(\xi_{0, t}(x)\right) \cdot \Xi_{0, t}(x) d W_{t}^{k} .\right.
$$

Therefore $\mu_{t}(d x)=Q_{t} \mu(d x)$ solves equation (1.2) in weak form, i.e.

$$
d \mu_{t}=L^{*} \mu_{t} d t+\sum_{k=1}^{d} B_{k}^{*} \mu_{t} d W_{t}^{k}, \quad \mu_{0}=\mu .
$$

Consider next the following two different assumptions on the original measure $\mu_{0}(d x)$ :

Assume that the original measure $\mu(d x)$ has a density $q(x)$ with respect to the Lebesgue measure on $\mathbf{R}^{m}$, i.e. $\mu(d x)=q(x) d x$. Then, the transformed measure $Q_{t} \mu(d x)$ has itself a density $q_{t}(x)$ which satisfies

$$
q_{t}\left(\xi_{0, t}(x)\right) \cdot J_{0, t}(x)=\Xi_{0, t}(x) \cdot q(x),
$$

or in integrated form

$$
\int_{A} q_{t}(x) d x=\int_{\xi_{0, t}^{-1}(A)} \Xi_{0, t}(x) \cdot q(x) d x .
$$

Here, $J_{0, t}(\cdot)$ is the Jacobian (i.e. the determinant of the Jacobian matrix) of the stochastic flow $\xi_{0, t}(\cdot)$. In addition, the density $q_{t}(x)$ solves the degenerate second order stochastic PDE

$$
d q_{t}=L^{*} q_{t} d t+\sum_{k=1}^{d} B_{k}^{*} q_{t} d W_{t}^{k}, \quad q_{0}=q .
$$

Assume that the original measure $\mu(d x)$ is a convex linear combination of Dirac masses, also called particles

$$
\mu(d x)=\sum_{i \in I} a^{i} \delta\left(x-x^{i}\right)
$$

where $\left\{a^{i}, i \in I\right\}$ are the particle weights, and $\left\{x^{i}, i \in I\right\}$ are the particle locations. Then, the transformed measure $Q_{t} \mu(d x)$ has a similar representation

$$
Q_{t} \mu(d x)=\sum_{i \in I} a_{t}^{i} \delta\left(x-x_{t}^{i}\right)
$$


where the particles have been transported by the flow i.e. $x_{t}^{i}=\xi_{0, t}\left(x^{i}\right)$, and the weights have been updated according to $a_{t}^{i}=a^{i} \Xi_{0, t}\left(x^{i}\right)$.

The idea behind particle approximation for equation (1.2) is the following :

- given an initial condition $\mu_{0}(d x)$ with density $q_{0}(x)$, find an approximation $\mu_{0}^{h}(d x)$ in terms of a linear convex combination of Dirac masses,

- use the exact solution of equation (1.4) with the approximation $\mu_{0}^{h}(d x)$ as initial condition, as an approximation for the solution of the original equation (1.5), and get error estimate if possible.

This can be illustrated by the following diagram

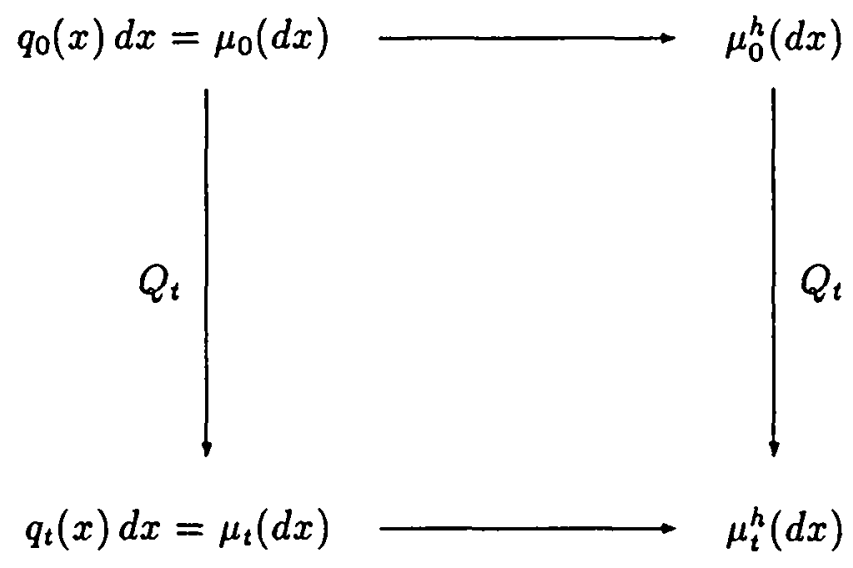

The remaining of this section is devoted to recalling standard results concerning stochastic flows of diffeomorphisms and stochastic PDE.

Proposition 1.1 Let $n \geq 0$ be fixed. Assume that

- $b, \sigma$ and $e$ have bounded derivatives up to order $(n+1)$,

- $c$ has bounded derivatives up to order $n$.

Then $\xi_{s, t}(\cdot)$ is a $C^{n}$-diffeomorphism in $\mathbf{R}^{m}$. In addition, the following estimates hold for all $p \geq 1$

$$
\begin{array}{ll}
\sup _{x \in \mathbb{R}^{m}} \mathbf{E}\left[\left|D^{\alpha} \xi_{s, t}(x)\right|^{p}\right]<\infty, & 1 \leq|\alpha| \leq n, \\
\sup _{x \in \mathbb{R}^{m}} \mathbf{E}\left[\left|D^{\alpha} \Xi_{s, t}(x)\right|^{p}\right]<\infty, & 0 \leq|\alpha| \leq n .
\end{array}
$$


Restricting to compact sets of $\mathbf{R}^{m}$, it is possible to invert the supremum and the mathematical expectation in the estimates above, see the Corollary 4.6.7 of Kunita [5]

Proposition 1.2 Under the assumptions of the Proposition 1.1, there exists a constant $C>0$, such that for any compact set $B \subset \mathbf{R}^{m}$ and $\varepsilon>0$ the following uniform estimates hold for all $p \geq 1$

$$
\begin{array}{ll}
\mathbf{E}\left[\sup _{x \in B}\left|D^{\alpha} \xi_{s, t}(x)\right|^{p}\right] \leq C\left[1+\delta^{p-\varepsilon}\right], & 1 \leq|\alpha| \leq n, \\
\mathbf{E}\left[\sup _{x \in B}\left|D^{\alpha} \Xi_{s, t}(x)\right|^{p}\right] \leq C\left[1+\delta^{p-\varepsilon}\right], & 0 \leq|\alpha| \leq n,
\end{array}
$$

where $\delta=\delta(B)$ denotes the diameter of $B$.

For all $n \geq 0, p \geq 1$, let $W^{n, p} \equiv W^{n, p}\left(\mathbf{R}^{m}\right)$ denote the space of real-valued Lebesguemeasurable functions on $\mathbf{R}^{m}$ whose generalized derivatives up to order $n$ are integrable in $p$-mean, and define the corresponding norm $\|\cdot\|_{n, p}$ and semi-norm $|\cdot|_{n, p}$ by

$$
\|u\|_{n, p}^{p} \triangleq \sum_{0 \leq|\alpha| \leq n} \int\left|D^{\alpha} u(x)\right|^{p} d x \quad \text { and } \quad|u|_{n, p}^{p} \triangleq \sum_{|\alpha|=n} \int\left|D^{\alpha} u(x)\right|^{p} d x \text {, }
$$

respectively.

Consider the following degenerate second order stochastic PDE

$$
d q_{t}=L^{*} q_{t} d t+\sum_{k=1}^{d} B_{k}^{*} q_{t} d W_{t}^{k}, \quad q_{0}=q .
$$

Although no coercivity hypothesis is satisfied, the following existence, uniqueness and regularity result is proved in Krylov-Rozovskii [2].

Theorem 1.3 Let $n \geq 1$ be fixed. Assume that

- $a$ has bounded derivatives up to order $\max (n, 2)$,

- $b, \sigma, c$ and $e$ have bounded derivatives up to order $n$,

- the initial condition satisfies $q_{0} \in W^{n, p}$.

Then equation (1.6) has a unique solution $q \in M^{p}\left(0, T ; W^{n, p}\right)$. In addition

$$
q \in L^{p}\left(\Omega ; C_{w}\left([0, T] ; W^{n, p}\right)\right),
$$

and the following estimate holds

$$
\mathbf{E}\left[\sup _{0 \leq t \leq T}\left\|q_{t}\right\|_{n, p}^{p}\right] \leq\left\|q_{0}\right\|_{n, p}^{p} e^{C T} .
$$




\section{Quadrature-based particle approximation}

With the quadrature formula (A.1)

$$
\int g(x) d x \sim \sum_{i \in I} \omega^{i} g\left(x^{i}\right)
$$

is associated the following particle approximation for the initial density $q_{0}(x)$

$$
q_{0}(x) d x=\mu_{0}(d x) \sim \mu_{0}^{h}(d x)=\sum_{i \in I} \omega^{i} q_{0}\left(x^{i}\right) \delta\left(x-x^{i}\right)
$$

This induces the following particle approximation for the solution $q_{t}(x)$ of equation (1.6)

$$
q_{t}(x) d x=\mu_{t}(d x) \sim \mu_{t}^{h}(d x)=\sum_{i \in I} \omega^{i} \Xi_{0, t}\left(x^{i}\right) q_{0}\left(x^{i}\right) \delta\left(x-\xi_{0, t}\left(x^{i}\right)\right)
$$

The following error estimate holds in Sobolev space with negative exponent, which extends the result of Raviart to the case of first order stochastic PDE.

Theorem 2.1 Let $n \geq m$ be fixed. Assume that

- $b, \sigma, c$ and e have bounded derivatives up to order $(n+1)$,

- the initial condition satisfies $q_{0} \in W^{n, p}$.

Then there exists a constant $C>0$ independent of $h$, such that

$$
\mathbf{E}\left\|\mu_{t}-\mu_{t}^{h}\right\|_{-n, p} \leq C h^{n}\left\|q_{0}\right\|_{n, p} .
$$

Proof. Let $\phi \in W^{n, p^{\prime}}$ be an arbitrary test function. Since

$$
\left\langle\mu_{t}, \phi\right\rangle=\int \phi\left(\xi_{0, t}(x)\right) \Xi_{0, t}(x) q_{0}(x) d x, \quad\left\langle\mu_{t}^{h}, \phi\right\rangle=\sum_{i \in I} \omega^{i} \phi\left(\xi_{0, t}\left(x^{i}\right)\right) \Xi_{0, t}\left(x^{i}\right) q_{0}\left(x^{i}\right),
$$

it follows from Theorem A.2 that

$$
\left|\left\langle\mu_{t}, \phi\right\rangle-\left\langle\mu_{t}^{h}, \phi\right\rangle\right| \leq C h^{n}|g|_{n, 1}
$$

with $g=\phi_{\circ} \xi_{0, t} \cdot \Xi_{0, t} q_{0}$, provided $g \in W^{n, 1}, n \geq m$.

Under the assumptions on the coefficients, $\phi \circ \xi_{0, t} \in W^{n, p^{\prime}}$ and $\Xi_{0, t} \cdot q_{0} \in W^{n, p}$, for conjugate $p$ and $p^{\prime}$. Moreover, the generalized Leibniz formula yields

$$
|g|_{n, 1} \leq \sum_{(\alpha, \beta) \in I_{n}} \int\left|\chi_{\alpha, \beta}(x) D^{\alpha} \phi\left(\xi_{0, t}(x)\right) D^{\beta} q_{0}(x)\right| d x
$$


where $I_{n}$ denotes the set of pairs $(\alpha, \beta)$ of multi-indices such that $|\alpha|+|\beta| \leq n$, and $\chi_{\alpha, \beta}(\cdot)$ are random fields involving the derivatives of $\xi_{0, t}(\cdot)$ and $\Xi_{0, t}(\cdot)$ up to order $n$. Using back and forth the changes of variable induced by the differomorphisms $\xi_{0, t}(\cdot)$ and $\xi_{0, t}^{-1}(\cdot)$, and the Hölder inequality, gives

$$
\begin{aligned}
|g|_{n, 1} & \leq \sum_{(\alpha, \beta) \in I_{n}} \int\left|\chi_{\alpha, \beta}\left(\xi_{0, t}^{-1}(x)\right) D^{\alpha} \phi(x) D^{\beta} q_{0}\left(\xi_{0, t}^{-1}(x)\right)\right|\left[J_{0, t}\left(\xi_{0, t}^{-1}(x)\right)\right]^{-1} d x \\
& \leq \sum_{(\alpha, \beta) \in I_{n}}\left\{\int\left|D^{\alpha} \phi(x)\right|^{p^{\prime}} d x\right\}^{1 / p^{\prime}}\left\{\int\left|\chi_{\alpha, \beta}\left(\xi_{0, t}^{-1}(x)\right) D^{\beta} q_{0}\left(\xi_{0, t}^{-1}(x)\right)\right|^{p}\right. \\
& \left.\left.\leq \| J_{0, t}\left(\xi_{0, t}^{-1}(x)\right)\right]^{-p} d x\right\}^{1 / p} \\
&
\end{aligned}
$$

Therefore

$$
\frac{\left|\left\langle\mu_{t}, \phi\right\rangle-\left\langle\mu_{t}^{h}, \phi\right\rangle\right|}{\|\phi\|_{n, p^{\prime}}} \leq C h^{n} \sum_{(\alpha, \beta) \in I_{n}}\left\{\int\left|\chi_{\alpha, \beta}(x) D^{\beta} q_{0}(x)\right|^{p}\left[J_{0, t}(x)\right]^{-(p-1)} d x\right\}^{1 / p}
$$

and

$$
\begin{array}{r}
\mathbf{E}\left\|\mu_{t}-\mu_{t}^{h}\right\|_{-n, p} \leq C h^{n} \sum_{(\alpha, \beta) \in I_{n}}\left\{\int \mathbf{E}\left\{\left|\chi_{\alpha, \beta}(x)\right|^{p}\left[J_{0, t}(x)\right]^{-(p-1)}\right\}\right. \\
\left.\left|D^{\beta} q_{0}(x)\right|^{p} d x\right\}^{1 / p}
\end{array}
$$

From estimates in Proposition 1.1, it holds

$$
\sup _{x \in \mathbb{R}^{m}} \mathbf{E}\left\{\left|\chi_{\alpha, \beta}(x)\right|^{p}\left[J_{0, t}(x)\right]^{-(p-1)}\right\}<\infty
$$

so that

$$
\mathbf{E}\left\|\mu_{t}-\mu_{t}^{h}\right\|_{-n, p} \leq C h^{n}\left\|q_{0}\right\|_{n, p}
$$

\section{Regularization}

Let $\zeta(x)$ be a continuous cut-off function defined on $\mathbf{R}^{m}$, which satisfies

$$
\begin{array}{ll}
\text { (i) } & \int \zeta(x) d x=1, \\
\text { (ii) } & \int x^{\alpha} \zeta(x) d x=0, \quad 1 \leq|\alpha| \leq k-1, \\
\text { (iii) } & \int|x|^{k}|\zeta(x)| d x<\infty,
\end{array}
$$


for some $k \geq 2$. For any $\varepsilon>0, \zeta_{\varepsilon}(x)$ is defined by the following scaling

$$
\zeta_{e}(x) \triangleq \frac{1}{\varepsilon^{m}} \zeta\left(\frac{x}{\varepsilon}\right)
$$

With the particle approximation

$$
\mu_{t}^{h}(d x)=\sum_{i \in I} \omega^{i} \Xi_{0, t}\left(x^{i}\right) q_{0}\left(x^{i}\right) \delta\left(x-x_{t}^{i}\right)
$$

is associated the regularized measure

$$
\mu_{t}^{h, \varepsilon}(d x)=\mu_{t}^{h} * \zeta_{\varepsilon}(d x)=q_{t}^{h, \varepsilon}(x) d x,
$$

where the density $q_{t}^{h, z}(x)$ is given by

$$
q_{t}^{h, \varepsilon}(x)=\sum_{i \in I} \omega^{i} \Xi_{0, \imath}\left(x^{i}\right) q_{0}\left(x^{i}\right) \zeta_{\ell}\left(x-x_{\mathfrak{t}}^{i}\right) .
$$

The main result of this section is the following theorem, which is an extension of the Theorem 4.2 in [6], to the case of first order stochastic PDE.

Theorem 2.2 Let $n>m$ be fixed. Assume that

- the cut-off function $\zeta$ satisfies (i)-(iii) for some $k \geq 2$, and $\zeta \in W^{n, 1}$,

- $b, \sigma, c$ and e have bounded derivatives up to order $(\ell+1)$,

- the initial condition satisfies $q_{0} \in W^{\ell, p}$,

where $\ell=\max (k, n)$.

Then, there exists a constant $C$ independent of both $h$ and $\varepsilon$, such that

$$
\left\{\mathbf{E}\left\|q_{t}-q_{t}^{h, \varepsilon}\right\|_{0, p}^{p}\right\}^{1 / p} \leq C\left\{\varepsilon^{k}\left\|q_{0}\right\|_{k, p}+(h / \varepsilon)^{n}\left\|q_{0}\right\|_{n, p}\right\} .
$$

Proof. Obviously

$$
q_{t}-q_{t}^{h, \varepsilon}=\left[q_{t}-q_{t} * \zeta_{\varepsilon}\right]+\left[q_{t} * \zeta_{\varepsilon}-q_{t}^{h, \varepsilon}\right] .
$$

First, it follows from Lemma 4.4 in [6] that

$$
\left\|q_{t}-q_{t} * \zeta_{e}\right\|_{0, p} \leq C \varepsilon^{k}\left|q_{t}\right|_{k, p}
$$

provided $q_{t} \in W^{k, p}$. Under the assumptions, Theorem 1.3 gives

$$
\left\{\mathbf{E}\left\|q_{t}-q_{t} * \zeta_{\varepsilon}\right\|_{0, p}^{p}\right\}^{1 / p} \leq C \varepsilon^{k}\left\{\mathbf{E}\left|q_{t}\right|_{k, p}^{p}\right\}^{1 / p} \leq C \varepsilon^{k}\left\|q_{0}\right\|_{k, p} .
$$


On the other hand, using the change of variable induced by the diffeomorphism $\xi_{0, \imath}^{-1}(\cdot)$, it holds for all $x \in \mathbf{R}^{m}$

$$
\begin{aligned}
q_{t} * \zeta_{e}(x)-q_{t}^{h, e}(x)=\int & \Xi_{0, t}(z) q_{0}(z) \zeta_{e}\left(x-\xi_{0, t}(z)\right) d z \\
& \quad-\sum_{i \in I} \omega^{i} \Xi_{0, t}\left(x^{i}\right) q_{0}\left(x^{i}\right) \zeta_{e}\left(x-\xi_{0, t}\left(x^{i}\right)\right)=E(g(x, \cdot))
\end{aligned}
$$

with $g(x, \cdot)=\Xi_{0, t} q_{0} \cdot \zeta_{\varepsilon}\left(x-\xi_{0, t}\right)$. Therefore, it follows from Theorem A.1 that for all $x \in \mathbf{R}^{m}$

$$
\left|q_{t} * \zeta_{\epsilon}(x)-q_{t}^{h, \varepsilon}(x)\right| \leq C h^{n}|g(x, \cdot)|_{n, 1}
$$

provided $g(x, \cdot) \in W^{n, 1}, n \geq m$. Moreover, the generalized Leibniz formula yields

$$
|g(x, \cdot)|_{n, 1} \leq \sum_{(\alpha, \beta) \in I_{n}} \int\left|\chi_{\alpha, \beta}^{\prime}(z) D^{\beta} q_{0}(z) D^{\alpha} \zeta_{\varepsilon}\left(x-\xi_{0, t}(z)\right)\right| d x
$$

where $I_{n}$ denotes the set of pairs $(\alpha, \beta)$ of multi-indices such that $|\alpha|+|\beta| \leq n$, and $\chi_{\alpha, \beta}^{\prime}(\cdot)$ are random fields involving the derivatives of $\xi_{0, t}(\cdot)$ and $\Xi_{0, t}(\cdot)$ up to order $n$. From the technical lemma below, it follows that

$$
\begin{gathered}
\int|g(x, \cdot)|_{n, 1}^{p} d x \leq C \sum_{(\alpha, \beta) \in I_{n}}\left\{\int\left|D^{\alpha} \zeta_{\varepsilon}(x)\right| d x\right\}^{p}\left\{\int\left|\chi_{\alpha \beta}^{\prime}(x) D^{\beta} q_{0}(x)\right|^{p}\right. \\
\left.\left[J_{0, t}(x)\right]^{-(p-1)} d x\right\}
\end{gathered}
$$

Making use of

$$
D^{\alpha} \zeta_{\varepsilon}(x)=\frac{1}{\varepsilon^{m+|\alpha|}} D^{\alpha} \zeta\left(\frac{x}{\varepsilon}\right)
$$

taking mathematical expectation on both sides, and raising to the power $1 / p$ gives

$$
\begin{array}{r}
\left\{\mathbf{E} \int|g(x, \cdot)|_{n, 1}^{p} d x\right\}^{1 / p} \leq C \frac{1}{\varepsilon^{n}}\|\zeta\|_{n, 1} \sum_{(\alpha, \beta) \in I_{n}}\left\{\int \mathbf{E}\left\{\left|\chi_{\alpha, \beta}^{\prime}(x)\right|^{p}\left[J_{0, t}(x)\right]^{-(p-1)}\right\}\right. \\
\left.\left|D^{\beta} q_{0}(x)\right|^{p} d x\right\}^{1 / p} .
\end{array}
$$

From estimates in Proposition 1.1, it holds

$$
\sup _{x \in \mathbf{R}^{m}} \mathbf{E}\left\{\left|\chi_{\alpha, \beta}^{\prime}(x)\right|^{p}\left[J_{0, t}(x)\right]^{-(p-1)}\right\}<\infty
$$

Therefore

$$
\begin{aligned}
\left\{\mathrm{E}\left\|q_{t} * \zeta_{e}-q_{t}^{h, \varepsilon}\right\|_{0, p}^{p}\right\}^{1 / p} & \leq C h^{n}\left\{\mathrm{E} \int|g(x, \cdot)|_{n, 1}^{p} d x\right\}^{1 / p} \\
& \leq C(h / \varepsilon)^{n}\|\zeta\|_{n, 1}\left\|q_{0}\right\|_{n, p} .
\end{aligned}
$$


Lemma 2.3 Let $f \in L^{p}$ and $g \in L^{1}$, and define

$$
I(x)=\int f(z) g\left(x-\xi_{0, t}(z)\right) d z
$$

Then $I \in L^{p}$ and in addition

$$
\left\{\int|I(x)|^{p} d x\right\}^{1 / p} \leq\left\{\int|f(x)|^{p}\left[J_{0, t}(x)\right]^{-(p-1)} d x\right\}^{1 / p} \int|g(x)| d x .
$$

PROOF. Using back and forth the changes of variable induced by the differomorphisms $\xi_{0, t}(\cdot)$ and $\xi_{0, t}^{-1}(\cdot)$, and the Lemma 4.3 in [6], gives

$$
I(x)=\int f\left(\xi_{0, t}^{-1}(z)\right)\left[J_{0, t}\left(\xi_{0, t}^{-1}(z)\right)\right]^{-1} g(x-z) d z
$$

and

$$
\begin{aligned}
\left\{\int|I(x)|^{p} d x\right\}^{1 / p} & \leq\left\{\int\left|f\left(\xi_{0, t}^{-1}(x)\right)\right|^{p}\left[J_{0, t}\left(\xi_{0, t}^{-1}(x)\right]^{-p} d x\right\}^{1 / p} \int|g(x)| d x\right. \\
& \leq\left\{\int|f(x)|^{p}\left[J_{0, t}(x)\right]^{-(p-1)} d x\right\}^{1 / p} \int|g(x)| d x
\end{aligned}
$$




\section{Adapted particle approximation}

Consider the particle approximation (A.3) for the initial condition $\mu_{0}(d x)$

$$
\mu_{0}(d x) \sim \mu_{0}^{h}(d x)=\sum_{i \in I} a^{i} \delta\left(x-x^{i}\right)
$$

where the particle weights $\left\{a^{i}, i \in I\right\}$ and the particle locations $\left\{x^{i}, i \in I\right\}$ are defined in the following way

$$
a^{i} \triangleq \mu_{0}\left(B^{i}\right)=\int_{B^{i}} \mu_{0}(d x), \quad x^{i} \triangleq \frac{1}{a^{i}} \int_{B^{i}} x \mu_{0}(d x)
$$

depending on the measure $\mu_{0}(d x)$. This induces the following particle approximation for the solution $\mu_{t}(x)$ of equation (1.4)

$$
\mu_{t}(d x) \sim \mu_{t}^{h}(d x)=\sum_{i \in I} a^{i} \Xi_{0, t}\left(x^{i}\right) \delta\left(x-\xi_{0, t}\left(x^{i}\right)\right)
$$

Parallel to the Theorem 2.1 above, the following error estimate holds in Sobolev space with negative exponent.

Theorem 3.1 Assume that

- $b, \sigma, c$ and e have bounded derivatives up to order 3 ,

- for all $i \in I$, the set $B^{i} \subset \mathbf{R}^{m}$ is compact.

Then there exists a constant $C>0$, such that

$$
\mathbf{E}\left\|\mu_{t}-\mu_{t}^{h}\right\|_{-2,1} \leq C \sum_{i \in I} \delta_{i}^{2} a^{i}
$$

where $a^{i}=\mu_{0}\left(B^{i}\right)$ and $\delta_{i}=\delta\left(B^{i}\right)$ denotes the diameter of the set $B^{i}$.

ProOF. Let $\phi \in W^{2, \infty}$ be an arbitrary test function. Since

$$
\left\langle\mu_{t}, \phi\right\rangle=\int \phi\left(\xi_{0, t}(x)\right) \Xi_{0, \ell}(x) \mu_{0}(d x), \quad\left\langle\mu_{t}^{h}, \phi\right\rangle=\sum_{i \in I} a^{i} \phi\left(\xi_{0, t}\left(x^{i}\right)\right) \Xi_{0, t}\left(x^{i}\right)
$$

it follows from estimate (A.6) that

$$
\left|\left\langle\mu_{t}, \phi\right\rangle-\left\langle\mu_{t}^{h}, \phi\right\rangle\right| \leq \frac{1}{2} \sum_{i \in I}|g|_{2, \infty, \widehat{B}^{i}} \delta_{i}^{2} a^{i}
$$


with $g=\phi \circ \xi_{0, t} \cdot \Xi_{0, t}$, where $\hat{B}^{i}$ denotes the convex hull of $B^{i}$. The generalized Leibniz formula yields

$$
\begin{aligned}
|g|_{2, \infty, B} & \leq \sum_{|\alpha| \leq 2} \sup _{x \in B}\left|\chi_{\alpha}(x) D^{\alpha} \phi\left(\xi_{0, t}(x)\right)\right| \\
& \leq \sum_{|\alpha| \leq 2}\left[\sup _{x \in B}\left|\chi_{\alpha}(x)\right|\right]\left[\sup _{x \in \mathbb{R}^{m}}\left|D^{\alpha} \phi(x)\right|\right] \\
& \leq\|\phi\|_{2, \infty} \sum_{|\alpha| \leq 2} \sup _{x \in B}\left|\chi_{\alpha}(x)\right|
\end{aligned}
$$

where $\chi_{\alpha}(\cdot)$ are random fields involving the derivatives of $\xi_{0, t}(\cdot)$ and $\Xi_{0, t}(\cdot)$ up to order 2 . Therefore

$$
\frac{\left|\left\langle\mu_{t}, \phi\right\rangle-\left\langle\mu_{t}^{h}, \phi\right\rangle\right|}{\|\phi\|_{2, \infty}} \leq \frac{1}{2} \sum_{i \in I} \sum_{|\alpha| \leq 2} \sup _{x \in \widehat{B}^{i}}\left|\chi_{\alpha}(x)\right| \delta_{i}^{2} a^{i}
$$

and

$$
\mathbf{E}\left\|\mu_{t}-\mu_{t}^{h}\right\|_{-2,1} \leq \frac{1}{2} \sum_{i \in I} \sum_{|\alpha| \leq 2} \mathbf{E}\left[\sup _{x \in \widehat{B}^{i}}\left|\chi_{\alpha}(x)\right|\right] \delta_{i}^{2} a^{i} .
$$

From estimates in Proposition 1.2, it holds

$$
\mathbf{E}\left[\sup _{x \in \hat{B}^{i}}\left|\chi_{\alpha}(x)\right|\right] \leq C\left[1+\delta_{i}^{2-\varepsilon}\right]
$$

for some $p$, where $\delta_{i}=\delta\left(B^{i}\right)$ denotes the diameter of both $B^{i}$ and its convex hull $\widehat{B}^{i}$, so that

$$
\mathbf{E}\left\|\mu_{t}-\mu_{t}^{h}\right\|_{-2,1} \leq C \sum_{i \in I}\left[1+\delta_{i}^{2-\varepsilon}\right] \delta_{i}^{2} a^{i}
$$




\section{References}

[1] P. FLORCHINGER and F. LE GLAND, Time-discretization of the Zakai equation for diffusion processes observed in correlated noise, Stochastics and Stochastics Reports 35 (4) 233-256 (1991).

[2] N.V. KRYLOV and B.L. ROZOVSKII, Characteristics of degenerating secondorder parabolic Itô equations, J.Soviet Math. 32 (4) 336-348 (1982).

[3] H. KUNITA, Stochastic partial differential equations connected with nonlinear filtering, in: Nonlinear Filtering and Stochastic Control (Cortona-1981) (eds. S.K.Mitter and A.Moro) 100-169, Springer-Verlag (LNM-972) (1982).

[4] H. KUNITA, First order partial differential equations, in: Stochastic Analysis (Katata and Kyoto-1982) (ed. K.Itô) 249-269, North-Holland (1984).

[5] H. KUNITA, Stochastic Flows and Stochastic Differential Equations, Cambridge University Press (1990).

[6] P.A. RAVIART, An analysis of particle methods, in: Numerical Methods in Fluid Dynamics (Como-1983) (ed. F.Brezzi) 243-324, Springer-Verlag (LNM1127) (1985). 


\section{A Particle approximation of functions}

Consider the following quadrature formula on $\mathbf{R}^{m}$

$$
\int g(x) d x \sim \sum_{i \in I} \omega^{i} g\left(x^{i}\right)
$$

where $\left\{x^{i}, i \in I\right\}$ is a coordinate grid of size $h>0, I=\mathrm{Z}^{m}$ and $\omega^{i}=h^{m}$ is the Lebesgue measure of the $m$-dimensional cube $B^{i}$ with center $x^{i}$ and edge size $h$. For all $g \in C\left(\mathbf{R}^{m}\right)$, the quadrature error associated with the quadrature formula (A.1) is defined by

$$
E_{i}(g) \triangleq \int_{B^{i}} g(x) d x-\omega^{i} g\left(x^{i}\right), \quad E(g) \triangleq \sum_{i \in I} E_{i}(g)
$$

The following estimate is proved in Raviart [6]

Theorem A.1 There is a constant $C>0$ independent of $h$ such that

$$
|E(g)| \leq C h^{n}|g|_{n, 1}
$$

for all $g \in W^{n, 1}, n \geq m$.

Let $\mu(d x)$ be a probability measure on $\mathrm{R}^{m}$ having a continuous density $q(x)$ with respect to the Lebesgue measure, i.e. $\mu(d x)=q(x) d x$. With the quadrature formula (A.1) is associated the following particle approximation for the density $q(x)$

$$
q(x) d x=\mu(d x) \sim \mu^{h}(d x)=\sum_{i \in I} \omega^{i} q\left(x^{i}\right) \delta\left(x-x^{i}\right),
$$

so that, for any test function $\phi$

$$
\langle\mu, \phi\rangle=\int \phi(x) q(x) d x, \quad\left\langle\mu^{h}, \phi\right\rangle=\sum_{i \in I} \omega^{i} \phi\left(x^{i}\right) q\left(x^{i}\right) .
$$

The following result is proved in Raviart [6]

Theorem A.2 There is a constant $C>0$ independent of $h$ such that

$$
\left\|\mu-\mu^{h}\right\|_{-n, p} \leq C h^{n}\|q\|_{n, p},
$$

for all $q \in W^{n, p}, n \geq m$.

Proof. From Theorem A.1, it holds

$$
\left|\langle\mu, \phi\rangle-\left\langle\mu^{h}, \phi\right\rangle\right|=|E(g)| \leq C h^{n}|g|_{n, 1},
$$


with $g=\phi \cdot q$, provided $g \in W^{n, 1}, n \geq m$. The generalized Leibniz formula and the Hölder inequality yield

$$
|g|_{n, 1} \leq C\|\phi\|_{n, p^{\prime}}\|q\|_{n, p}
$$

for conjugate $p$ and $p^{\prime}$, and therefore

$$
\left\|\mu-\mu^{h}\right\|_{-n, p}=\sup _{\phi \in W^{n, p^{\prime}}} \frac{\left|\langle\mu, \phi\rangle-\left\langle\mu^{h}, \phi\right\rangle\right|}{\|\phi\|_{n, p^{\prime}}} \leq C h^{n}\|q\|_{n, p} .
$$

Another possible approximation is to consider a partition $\left\{B^{i}, i \in I\right\}$ of $\mathbf{R}^{m}$, and to define the following particle approximation for the probability measure $\mu(d x)$

$$
\mu(d x) \sim \mu^{h}(d x)=\sum_{i \in I} a^{i} \delta\left(x-x^{i}\right),
$$

where the particle weights $\left\{a^{i}, i \in I\right\}$ and the particle locations $\left\{x^{i}, i \in I\right\}$ are defined in the following way

$$
a^{i} \triangleq \mu\left(B^{i}\right)=\int_{B^{i}} \mu(d x), \quad x^{i} \triangleq \frac{1}{a^{i}} \int_{B^{i}} x \mu(d x)
$$

depending on the measure $\mu(d x)$ so that, for any test function $\phi$

$$
\langle\mu, \phi\rangle=\int \phi(x) \mu(d x), \quad\left\langle\mu^{h}, \phi\right\rangle=\sum_{i \in I} a^{i} \phi\left(x^{i}\right) .
$$

For all $\phi \in C\left(\mathbf{R}^{m}\right)$, the quadrature error associated with the formula (A.3), is defined by

$$
E_{i}^{\prime}(\phi) \triangleq \int_{B^{i}} \phi(x) \mu(d x)-a^{i} \phi\left(x^{i}\right), \quad E^{\prime}(\phi) \triangleq \sum_{i \in I} E_{i}^{\prime}(\phi) .
$$

Parallel to the Theorem A.2 above, the following result holds

Theorem A.3 For any partition $\left\{B^{i}, i \in I\right\}$

$$
\left\|\mu-\mu^{h}\right\|_{-2,1} \leq \frac{1}{2} \sum_{i \in I} \delta_{i}^{2} a^{i}
$$

where $a^{i}=\mu\left(B^{i}\right)$ and $\delta_{i}=\delta\left(B^{i}\right)$ denotes the diameter of the set $B^{i}$.

Proof. Let $\phi \in W^{2, \infty}$ be an arbitrary test-function. Using Taylor expansion around the point $x=x^{i}$ yields

$$
\begin{aligned}
\phi(x)= & \phi\left(x^{i}\right)+\left(x-x^{i}\right)^{*} D \phi\left(x^{i}\right) \\
& +\left(x-x^{i}\right)^{*}\left\{\int_{0}^{1}(1-u) D^{2} \phi\left[u x+(1-u) x^{i}\right] d u\right\}\left(x-x^{i}\right),
\end{aligned}
$$


and the definition (A.4) gives

$$
E_{i}^{\prime}(\phi)=\int_{B^{i}}\left(x-x^{i}\right)^{*}\left\{\int_{0}^{1}(1-u) D^{2} \phi\left[u x+(1-u) x^{i}\right] d u\right\}\left(x-x^{i}\right) d x .
$$

Therefore

$$
\left|E_{i}^{\prime}(\phi)\right| \leq \frac{1}{2}|\phi|_{2, \infty, \hat{B}^{i}} \int_{B^{i}}\left\|x-x^{i}\right\|^{2} \mu(d x) \leq \frac{1}{2}|\phi|_{2, \infty, \widehat{B}^{i}} \delta_{i}^{2} a^{i},
$$

where $\hat{B}^{i}$ denotes the convex hull of $B^{i}$. Then

$$
\left|\langle\mu, \phi\rangle-\left\langle\mu^{h}, \phi\right\rangle\right|=\left|E^{\prime}(\phi)\right| \leq \frac{1}{2} \sum_{i \in I}|\phi|_{2, \infty, \widehat{B}^{i}} \delta_{i}^{2} a^{i},
$$

and

$$
\left\|\mu-\mu^{h}\right\|_{-2,1}=\sup _{\phi \in W^{2, \infty}} \frac{\left|\langle\mu, \phi\rangle-\left\langle\mu^{h}, \phi\right\rangle\right|}{\|\phi\|_{2, \infty}} \leq \frac{1}{2} \sum_{i \in I} \delta_{i}^{2} a^{i}
$$

Remark A.4 If the partition $\left\{B_{i}, i \in I\right\}$ is given, with $\delta_{i} \leq C h$ for all $i \in I$, then

$$
\left\|\mu-\mu^{h}\right\|_{-2,1} \leq C h^{2} \text {. }
$$

On the other hand, if the partition $\left\{B_{i}, i \in I\right\}$ has to be chosen so as to make the quadrature error as small as possible, then estimate (A.5) can be used to derive the following criterion

$$
\delta_{i}^{2} a^{i}=c \quad \text { for all } i \in I .
$$

This criterion based on equidistribution of the local quadrature error, has the following interesting property

- a set with a large mass, will be split into some smaller subsets,

- conversely, neighbouring sets with small masses, will be packed together into one single set. 
ISSN 0249 - 6399 